\title{
¿Estudios Organizacionales en Chile? Entre la parcelación, la ambigüedad y las racionalidades en disputa
}

\section{Organization Studies in Chile? Between fragmentation, ambiguity and disputing rationalities}

\author{
Nicolás Alejandro Ríos González ${ }^{1 *}$, Juan Pablo Toro², Gregorio Pérez-Arrau ${ }^{3}$, Marcela Mandiola Cotroneo ${ }^{4}$, \\ Alvaro Espejo ${ }^{5}$ \\ 1 Universidad de Barcelona, Barcelona, España \\ 2 Facultad de Psicología, Universidad Diego Portales, Santiago, Chile \\ 3 Facultad de Administración y Economía, Universidad de Santiago de Chile, Santiago, Chile \\ 4 Facultad de Economía y Negocios, Universidad Alberto Hurtado, Santiago, Chile \\ 5 Escuela de Negocios, Universidad Adolfo Ibáñez, Santiago, Chile \\ *nico.rios.g@gmail.com
}

Recibido: 9-junio-2018

Aceptado: 26-octubre-2018

\section{RESUMEN}

Este artículo busca aportar al debate en Chile respecto al desarrollo de un campo abarcativo e interdisciplinar como es el de los Estudios Organizacionales. Para ello se recorre la trayectoria de este campo desde su surgimiento en el norte global hasta sus desplazamientos por Latinoamérica, arribando a los hitos fundacionales que marcan el desarrollo del pensamiento organizacional en Chile. En este contexto, el artículo trae a escena las voces de diferentes académicos, investigadores y consultores organizacionales a partir de un diálogo generativo usando la metodología del world café. Tras realizar un análisis temático del contenido, los resultados permiten develar los principales desafíos situados que acarrea la constitución de un campo de identidad incierta como los estudios organizacionales: la parcelación disciplinaria, la ambigüedad identitaria y las racionalidades en disputa.

Palabras clave: administración, Chile, Estudios organizacionales, neoliberalismo

\section{ABSTRACT}

This article seeks to offer some contributions to the debate around the development of the field of organization studies in Chile. We trace the history of this field from its inception in the global north through its influence and shiftings around Latin America, recording the milestones of organizational thought in Chile. In this context, the paper provides opinions from various scholars, researchers and organizational consultants through a generative dialogue using the world cafe methodology. From a thematic content analysis, the results shed light on the major challenges around the establishment of a field such as organization studies in Chile, whose identity remains unclear: the fragmentation of disciplines, the field's ambiguity and its conflicting rationalities.

Keywords: Chile, management, neoliberalism, Organization studies

Cómo citar este artículo: Ríos González, N., Toro, J. P., Pérez-Arrau, G., Mandiola Cotroneo, M., \& Espejo, A. (2018). ¿Estudios Organizacionales en Chile? Entre la parcelación, la ambigüedad y las racionalidades en disputa. Psicoperspectivas, 17(3), 1-14.

http://dx.doi.org/10.5027/psicoperspectivas-vol17-issue3-fulltext-1389 
El Norte global como emplazamiento de los Estudios Organizacionales

Los orígenes de los Estudios Organizacionales (EO) pueden ubicarse en relación a las profundas transformaciones que generó la revolución industrial primariamente en el norte global, condición que trajo aparejado el interés intelectual por asuntos como el poder y las estructuras de dominación, la alienación obrera, la burocracia y el control social. Es en este contexto de crecimiento fabril, transformación social y cambio tecnológico en el cual se gestan obras de pensadores como Henri de Saint-Simon, Karl Marx, Émile Durkheim y Max Weber, quienes intentaron aprehender las nuevas configuraciones del fenómeno organizacional, razón por la cual sus trabajos suelen ser reconocidos como germinales en el campo del pensamiento organizacional.

Sin embargo, las sucesivas guerras y la migración de intelectuales que marcaron la primera mitad del siglo XX trasladó el eje del pensamiento en torno a lo organizacional desde Europa a Norteamérica, posicionando a Estados Unidos como un nuevo contexto de referencia. La producción académica sobre las organizaciones adoptó allí un marcado carácter individualista y orientado al crecimiento económico (Barba, 2012; Gonzáles-Miranda, 2014), en concordancia con el nuevo contexto al que respondía la denominada Teoría Organizacional (TO).

Si bien los estragos de la segunda guerra dificultaron el desarrollo académico del pensamiento organizacional proveniente de Europa, este tomó un nuevo impulso a partir de la década de los setenta del siglo XX. Esto implicó la emergencia de nuevas perspectivas sobre la organización y el proceso de organizar a la luz de debates en que convergieron cuestiones políticas y epistémicas tales como el antiimperialismo, el feminismo, los movimientos antirracistas y el giro decolonial, los desarrollos de la teoría crítica en las ciencias sociales, el postestructuralismo y la crítica postmoderna (March 2007). Será hacia los años noventa que lo anterior madurará gestando a los EO como un movimiento heterodoxo, crítico del positivismo imperante, y que ofrece nuevas perspectivas y problematizaciones que no se reducen a las categorías aportadas predominantemente por la teoría marxista (Misoczky, 2017).

Puede afirmarse que las rutas seguidas desde los orígenes de pensamiento organizacional hasta la gestación de los EO, sometidas a curvaturas históricas y diversos contextos geopolíticos de desarrollo, desembocan en una multiplicidad de adscripciones teórico-epistemológicas configurando un campo cuya única característica distintiva que no es cuestionada es precisamente su diversidad (March, 2007; de Faria, 2009). En este contexto, autores como Salgado (2007) y Reed (2017) han contribuido con sistematizaciones que permiten diferenciar las miradas predominantes dentro de las que se ubica esta diversidad. De este modo, señalan miradas donde el centro lo ocupa la preocupación por el orden y la eficiencia en los sistemas de trabajo, el consenso social en las organizaciones, las libertades individuales y la adaptabilidad evolutiva a la organización, el conocimiento y los mecanismos de poder, la justicia, la ética y la complejidad en las organizaciones.

En medio de esta rica pero a la vez confusa multiplicidad de perspectivas es posible identificar un lento pero sostenido distanciamiento entre miradas a lo organizacional donde por una parte predomina el racionalismo funcionalista de orientación eficientista $y$, por otra, una orientación comprensiva y más cercana a las ciencias sociales (Salgado, 2007; Gonzales-Miranda, 2014). La predominancia de estas perspectivas coincide con racionalidades distintas sobre lo organizacional, las que se muestran potencialmente transversales a los diferentes compromisos disciplinares de los EO. De este modo, este campo se va desarrollando en una confusa intersección de contenidos (Barba, 2012) con aportes de la administración, la psicología, la sociología, la historia, la filosofía, la antropología, la biología y la física (RamírezMartínez, Vargas, \& De la Rosa, 2011).

Como se puede evidenciar hasta aquí, la gestación y el dominio sobre el debate en torno a lo organizacional se ha concentrado en las esferas intelectuales estadounidenses y europeas, en coherencia con las condiciones históricas y situadas de ambos territorios. Sin embargo, siendo este debate un punto de gestación sobre el pensamiento organizacional, cabe la pregunta por su diseminación universalista, considerando la casi inexistente participación de y alusión a los países del sur, específicamente los latinoamericanos. Esta pregunta se vuelve relevante dada la multiplicidad que caracteriza el campo del pensamiento organizacional, toda vez que su expansión desde lo que hemos denominado 'norte global' podría no aprehender especificidades de otros territorios marginales a dicho eje geopolítico. Lo anterior se refuerza por el hecho que la génesis de los EO, así como su inacabado proceso de consolidación, ha estado caracterizado por una profusa y mayoritaria producción literaria en idioma inglés, con menores aportes en idiomas como el francés, el japonés o el castellano (Barba, \& Ramírez, 2004; Barba 2012). Por esta razón, resulta pertinente interrogarse por las formas particulares que el campo experimentó en territorios diferentes al norte global, especialmente en la región latinoamericana. 
Desplazamientos y dificultades de los Estudios Organizacionales en Latinoamérica

A lo largo del desarrollo de los EO en el norte global, ha existido una escasa cuando no nula referencia a los desarrollos del pensamiento en torno a lo organizacional en Latinoamérica. De acuerdo a Chumbita (2015) los procesos históricos y sociales de los países latinoamericanos han sido considerados como epifenómenos de los propios procesos de desarrollo de los países del norte global. Esto ha conducido a que Latinoamérica aparezca como punto ciego de la investigación global sobre organizaciones, al no llamar la atención de los grandes centros de estudio internacionales (Özbilgin, 2004; Nicholls-Nixon, et al. 2011) conduciendo a la escasa publicación y difusión del debate regional (Padilla, Hernández, \& Ríos, 2015).

Lo anterior ocurre a pesar de la reivindicación que desde los años 90 en adelante diversos agentes comenzaron a realizar sobre de la existencia de formas de organización alternativa en la región, las que abrazarían visiones, medios y fines basados en la colaboración, la participación, la comunidad y la horizontalidad (IbarraColado, 2006; Barba, 2012; Sanabria, Saavedra, \& Smida, 2013). En este contexto, si bien se han ido generando proyectos que recuperan el conocimiento propio sobre el quehacer organizativo en Latinoamérica, estos no se han desarrollado sin dificultades.

Una de las primeras dificultades concierne a la configuración de las prácticas y comunidades de investigación latinoamericanas asociados al desarrollo del pensamiento organizacional como campo de estudio. Además de ser una comunidad pequeña, débilmente articulada a nivel regional y con escasos recursos para el desarrollo de sus investigaciones, existen fuertes controversias y divisiones entre grupos que luchan por defender sus propias posiciones teóricas y epistemológicas, muchas veces en desmedro del diálogo constructivo (Pérez Arrau, Eades, \& Wilson, 2012; Padilla et al., 2015). Por esta razón, algunos han señalado la existencia de un anarquismo epistemológico en referencia a una diversidad paralizante que tiende a envolver a este campo (Medina, 2010).

Como reverso de lo anteriormente descrito, la investigación e intervención organizacional en Latinoamérica ha sido hegemonizada por administradores agrupados en asociaciones profesionales y académicas que promueven concepciones, literatura y teorías organizacionales inspiradas en modelos de gestión para fines estrictamente productivos. Generalmente estos modelos provienen de países ajenos a la realidad Latinoamericana y son introducidos sin mediar reflexión alguna respecto al contexto en que son aplicados, generando angustia, conflicto, fatalismo o el comportamiento hipócrita de quienes los adoptan sin creer en sus postulados y fundamentos (Chumbita, 2015; Gomez, \& Rodríguez, 2006; Hojman, \& Pérez Arrau, 2005).

Una segunda dificultad se relaciona con la progresiva y acentuada bifurcación entre las disciplinas de la administración y las ciencias sociales en lo que respecta a los desarrollos del pensamiento organizacional en la región. En parte, esta bifurcación bebe del distanciamiento entre las miradas organizacionales de corte funcionalista o comprensiva descritas previamente, las que se extenderían a través de múltiples territorios disciplinarios que nutren el campo. Sin embargo y en caso de Latinoamérica, este distanciamiento epistémico y disciplinar también puede ser explicado por la historia de una academia militante y crítica de la sociedad y su modelo de desarrollo al ritmo de las dictaduras cívicomilitares que posicionaron a las ciencias sociales como objeto de sospecha (Garretón, 2013).

Tanto la bifurcación entre ciencias sociales y administración como la captura del pensamiento organizacional por las disciplinas de la administración han conducido a una difícil adscripción al campo de los EO entre quienes trabajan en asuntos que podrían ser apuntados como propios de él. A pesar que se ha impulsado la existencia de estudios organizacionales en proximidad con las ciencias sociales y en relación con el acervo organizacional latinoamericano, lo organizacional continúa experimentando un estigma al interior de las ciencias sociales, dada su vinculación con una racionalidad administrativa e irreflexiva que ha sido asociada a un pragmatismo paralizante (Ibarra-Colado, \& Montaño, 1992, p. 17).

Una tercera y última dificultad puede relacionarse con el protagonismo que han jugado las parcelas disciplinarias al momento de aprehender los fenómenos organizacionales en Latinoamérica. Desde disciplinas consolidadas como la sociología, la psicología o la economía, los EO no ofrecen aportes significativos para la comprensión de problemas o el desarrollo de enfoques con sentido para la región, siendo ocasionalmente acusados de responder a intereses reaccionarios de las grandes corporaciones y el lucro (Salgado, 2007, p. 12). En este sentido, el pensamiento organizacional disciplinado sigue jugando un papel central como condición que contribuye a dificultar la configuración heterodoxa del campo en Latinoamérica.

Las dificultades anteriormente descritas están detrás de 
la ausencia y postergación de un debate situado que, simultáneamente, supone la negación de las propias virtudes y defectos de la región en lo que concierne a sus organizaciones y modos de organizar, perpetuando una situación de dependencia estructural, ideológica y epistemológica que poco contribuye a su desarrollo y autonomía (Ibarra-Colado, \& Montaño, 1992, de Faria, 2009). Adicionalmente, el escaso interés de las ciencias sociales sobre la administración como objeto de estudio (Medina, 2010) ha reforzado un desarrollo incipiente, fragmentario y de bajo impacto sobre las prácticas organizacionales.

No obstante lo anterior y a pesar del escenario descrito, es posible reconocer algunos aportes fundamentales al desarrollo de los EO en la región que han contribuido a visibilizar y legitimar este campo en su carácter transdisciplinario y heterodoxo en diversas latitudes. En ese sentido es imposible no referirse a países como México, Colombia y Brasil, destacando los aportes fundacionales de Ibarra-Colado y Montaño (1987, 1989, 1991, 1992) en el primero de estos. A ello se suman organizaciones especializadas que se han consolidado en dichos países (Padilla, et al., 2015; Gonzales-Miranda, Ocampo-Salazar, \& Gentilin, 2018), tales como la Red Mexicana de Investigadores en Estudios Organizacionales (REMINEO), la Red de Estudios Organizacionales Colombiana (REOC) y la Sociedad Brasileña de Estudios Organizacionales (SBEO). Cabe precisar igualmente la emergencia de nuevas agrupaciones y núcleos de estudio en países del sur como el Grupo Chileno de Estudios Organizacionales MINGA y el Grupo de Sociología del Management en Argentina.

A nivel internacional también se han fortalecido redes y eventos académicos de encuentro y discusión tales como La Red de Posgrados de Investigación Latinos en Administración y Estudios de la Organización (PILARES) y el Encuentro Europeo y Latinoamericano de Estudios Organizacionales (LAEMOS). Lo anterior ha ido de la mano de un creciente número de universidades que han creado programas de doctorado en este campo, los que inevitablemente abogan por más y mejor investigación en torno a lo organizacional, así como al desarrollo de redes internacionales (Barba, \& Ramírez, 2004; Padilla, et al., 2015). Así la evidencia indica que los estudios organizacionales avanzan sostenidamente hacia una posición de mayor relevancia en el debate académico y profesional sobre las organizaciones en Latinoamérica. Sin embargo, surge la pregunta por la especificidad de la experiencia chilena en relación al pensamiento organizacional, sus configuraciones disciplinarias en la academia nacional, la producción teórica en este campo y su lugar en medio de esta incesante e inacabada articulación de perspectivas, debates, redes y encuentros académicos a nivel internacional y latinoamericano.

Una visión sinóptica de los Estudios Organizacionales en Chile

Para indagar en los derroteros del pensamiento organizacional en Chile resulta útil conducirse históricamente por los contextos y condiciones de posibilidad que explican el surgimiento, permanencia o desaparición de sus diversas corrientes. En este sentido, cabe mencionar la trayectoria desarrollista que tomó lugar en la sociedad chilena, culminando con el gobierno de la Unidad Popular, trayectoria que fue interrumpida por el golpe de estado y el equipo económico de la dictadura militar. A partir de allí, los años 80 en Chile constituyen un periodo de referencia obligado para dar cuenta de un conjunto de transformaciones sociales, políticas y económicas del pasado reciente, las que se inauguran en dicha década del siglo XX y sobre las que existe relativa continuidad en los años venideros, impactando en el pensamiento organizacional y las organizaciones mismas. En el centro de estas transformaciones se ubica la consolidación de un modelo económico y productivo radicalmente neoliberal, instalado al alero de la dictadura militar y moderadamente ajustado por los gobiernos democráticos que le sucedieron.

Para efectos de lo que nos interesa debatir aquí, el proceso modernizador que se instala al calor del neoliberalismo trae con sí una importante reestructuración productiva de libre mercado, junto al auge de las privatizaciones y el predominio de la racionalidad competitiva, condiciones determinantes para el impulso analítico, comprensivo y crítico que experimentaron las ciencias sociales en este período y que acabaron por configurar una brecha entre éstas y el mundo de la empresa y la administración (Moulian, 1997). Como se expusiera previamente, esta bifurcación disciplinaria va de la mano de un distanciamiento de racionalidades y encuentra paralelismos con los procesos vividos en otras latitudes del subcontinente latinoamericano.

En este escenario, algunos hitos relevantes pueden ser señalados desde una visión sinóptica sobre el devenir histórico del pensamiento organizacional en Chile, especialmente destacando los aportes de instituciones y personas específicas que han jugado un rol protagónico en su desarrollo. Cabe destacar que esta revisión alude específicamente a contribuciones del ámbito académico, 
por tanto de naturaleza teórica y disciplinaria ${ }^{1}$, las que se articulan configurando formas específicas y particulares de lo que hemos optado por denominar como 'pensamiento organizacional'. Esto último, con el fin de evitar cualquier reificación de un campo de estudio al que, a nuestro juicio, estos antecedentes contribuyen pero sin necesariamente adscribirse a él.

Como primer foco de esta mirada sinóptica cabe mencionar el trabajo desplegado por más de 60 años en la actual Facultad de Economía y Negocios (FEN) de la Universidad de Chile. Su trayectoria ha sido caracterizada por Acuña (2017) como oscilante, discontinua, con cambios de foco y de perspectiva, en consonancia con las transformaciones del país, las instituciones y la propia universidad. El mismo autor identifica distintos momentos del recorrido de esta Facultad, caracterizados por acentos diferenciales en su trabajo académico. Ejemplo de ello es el foco puesto en las relaciones laborales entre los años 50 y 70 del siglo XX, así como la teoría de sistemas que durante los años 70 Oscar Johansen introdujo como modelo idóneo para el análisis y conceptualización del fenómeno organizacional (Johansen, 1971, 1980, 1989; Johansen, de la Cuadra, \& García, 1992).

Posteriormente y bajo el periodo de la dictadura se funda el Departamento de Relaciones Laborales y Desarrollo Organizacional (DERTO), espacio desde el cual se introduce el Desarrollo Organizacional y, más adelante, la Gestión de Recursos Humanos (Reyes, 2016). Cabe destacar que el modelo de recursos humanos es más liberal que la tradicional administración de personal. Esto se expresa en su declarado enfoque estratégico, su orientación hacia la flexibilidad, la búsqueda del compromiso de los empleados, un mayor involucramiento de los puestos de supervisión en la línea productiva y en las decisiones sobre la fuerza laboral y; por último, en la visión unilateral de las relaciones laborales en que se tiende a ignorar el papel de los sindicatos en las empresas (Guest, 1987). De este modo, se puede afirmar que la creación del DERTO en una Facultad que inicialmente detentaba una mirada pluralista de lo organizacional desde el DO y las relaciones laborales, va paulatinamente orientándose hacia una mirada productivista, funcionalista y tecnocrática dentro del pensamiento organizacional, perspectiva que ha devenido hegemónica, configurando un cuadro que perdura hasta la fecha en el escenario nacional.

\footnotetext{
${ }^{1}$ Se ha decidido excluir el acervo que proviene de la práctica profesional de la consultoría, un campo de acción e intervención en organizaciones que ha contribuido a enriquecer el pensamiento organizacional, a pesar de su escasa formalización y difícil acceso como publicaciones.
}

La creación del DERTO y la introducción del modelo de recursos humanos se refuerza por dos hechos. El primero de ellos dice relación con la llegada al país de jóvenes becados por el Estado, recientemente graduados en universidades norteamericanas en programas de estudio sobre materias laborales de enfoque neoliberal. El segundo consiste en la creación de cursos y posteriormente programas de estudio en universidades nacionales que asumieron el modelo de administración de recursos humanos (Pérez-Arrau, et al., 2012). Este escenario permite que la perspectiva de gestión de recursos humanos haya ido consolidándose como una corriente principal de pensamiento organizacional, desplazando paulatinamente los cursos tradicionales de relaciones laborales, economía laboral, teoría de las organizaciones y en definitiva relegando a los márgenes estas corrientes de pensamiento organizacional.

Al margen de lo anterior, cabe destacar el vínculo que el Departamento de Administración de la FEN estableció tempranamente desde los 90 con el Instituto Tavistock de Londres, lo que fue otorgando una impronta crítica a la perspectiva teórica y de intervención que ha desarrollado el departamento lo que, a nuestro juicio, abre una línea de influencia de la corriente de EO europeos en el país. De este modo, actualmente se reconocen en su trabajo dos enfoques: uno funcionalista y cuantitativo con orientación a la administración y otro cualitativo y con orientación a la dinámica organizacional desde la perspectiva teórica del socioanálisis y el management crítico.

Un segundo foco de esta mirada sinóptica apunta al trabajo desarrollado por Nicolás Majluf en el ámbito de la gestión empresarial desde la Escuela de Administración de la Universidad Católica de Chile. En 1996 introduce en el país, en un libro en coautoría con Arnoldo Hax, los principios y metodología de la gestión estratégica, editado originalmente en Estados Unidos en 1984. Más adelante, en 2011, publica "Los desafíos de la gestión: de lo formal a lo sutil", teniendo ambos textos un fuerte impacto en el ámbito académico y profesional de la administración, tanto por proponer un modelo propio e integrador de la perspectiva estratégica como por el carácter situado de sus aportes (Hax, \& Majluf, 1996; Majluf, 2011).

Complementando lo anterior, un tercer foco considera las contribuciones al pensamiento organizacional

Consideramos que una compilación e indagación en dicho trabajo merecería un artículo en sí mismo y excede los propósitos de este trabajo. 
realizadas por Darío Rodríguez, autor de textos de teoría, diagnóstico y gestión organizacional de amplia difusión, incluyendo lecturas obligatorias en la formación de profesionales del ámbito de la ingeniería y las ciencias sociales. Discípulo de Niklas Luhmann y catalizador de los aportes de Francisco Varela y Humberto Maturana, su trabajo ha contribuido a la comprensión de las organizaciones como sistemas complejos y autorreferidos, poniendo énfasis en las articulaciones entre sociedad y organización, con particular atención sobre los fenómenos organizacionales en la realidad latinoamericana y chilena (Abarca, Majluf, \& Rodríguez, 1998; Rodríguez, 1991, 1992, 2004; Rodríguez, \& Arnold, 1991; Rodríguez, Bozzo, \& Arnold, 1999; Rodríguez, \& Opazo, 2007).

Finalmente, una última contribución por enfocar consiste en el trabajo de Ramos (2009) sobre la transformación modernizadora que ha experimentado la empresa chilena en las últimas décadas. Su estudio identifica el carácter desbalanceado del postfordismo de la empresa chilena, en la que conviven la flexibilidad, la maleabilidad estratégica y una visión globalizante, pero todo ello en un escenario de relaciones internas marcadas por fuerte verticalismo, alto diferencial de poder y desconfianza. $\mathrm{Si}$ bien este estudio está limitado a empresas y no incluye otro tipo de organizaciones, sus conclusiones bien pueden extrapolarse a otros entornos. En este sentido, cabe mencionar otras contribuciones realizadas en una línea similar por investigadores que han caracterizado las dimensiones de la cultura organizacional prevalecientes en el país, así como las prácticas de recursos humanos asociadas a ellas (Hojman, \& Pérez-Arrau, 2005; Gómez, \& Rodríguez, 2006; Pérez-Arrau, Eades, \& Wilson, 2012; Rodríguez, \& Gómez, 2009).

Como se aprecia de esta visión sinóptica, el pensamiento organizacional en Chile transcurre por condiciones políticas, académicas y disciplinarias que van consolidando una perspectiva y corriente dominante, en contraposición al desarrollo de miradas alternativas frente al fenómeno organizacional, las que comienzan a emerger junto al trabajo de personas específicas hacia inicios de los años 90 . A nuestro juicio, las condiciones que desde ese entonces se abren permiten la emergencia de una heterodoxia en el pensamiento organizacional, de la mano de nuevas tendencias en la investigación, intervención y enseñanza. Siguiendo a Gonzales-Miranda et al. (2018), estas tendencias se aproximan a lo que puede entenderse como Estudios Organizacionales, lo que se refleja en los nuevos problemas y objetos de estudio que son adoptados en torno y a propósito de la organización, principalmente hacia inicios de los años 2000.
Dentro de estas nuevas tendencias se pueden señalar las perspectivas críticas en torno al management y la administración realizado por académicos y académicas en algunas escuelas de negocios, administración y ciencias sociales (Acuña, 2012; Espinoza, \& Rivera, 2015; Mandiola, 2013, 2017, 2018; Mandiola, Imas, \& Ascorra, 2014; Ramírez, 2003; Sisto, 2012; Subiabre, 2012). Lo crítico en estas miradas ha tenido estrecha relación con el posicionamiento epistémico-político de cada uno de los trabajos, incluyendo perspectivas discursivas (Ascorra, 2003; Mandiola, \& Ascorra, 2010), decoloniales (Mandiola, 2010, 2018) y de género (Ríos, Mandiola, \& Varas, 2017).

En intersección con la mirada organizacional pero con un foco en trabajo, otra línea de investigación a mencionar es la de subjetividad e identidades (Sisto, 2004; Soto, 2008; Soto, 2014; Stecher, 2014; Stecher, \& Godoy, 2014) en ámbitos como la política educativa (Assaél, Cornejo, González, Redondo, Sánchez, \& Sobarzo, 2011; Cornejo, Castañeda, \& Acuña, 2014; Sisto, Fardella, Ahumada, \& Montecinos, 2009) y las universidades (Fardella, Sisto, \& Jiménez, 2017; Sisto, 2005). A ello se suman líneas de trabajo en torno a la salud (Acuña, \& Sanfuentes, 2011, 2016; Sanfuentes, \& Acuña, 2014) y lo que se ha denominado clínicas del trabajo (Zabala, Guerrero, \& Besoaín, 2016) y malestar en el trabajo (Acuña, \& Silva, 2008; Foladori, \& Guerrero, 2018). Dentro de este ámbito cabe señalar igualmente las relaciones laborales y las organizaciones sindicales en el marco económico político del neoliberalismo (Julián, 2018a, 2018b).

En los contextos de desarrollo y debate internacional y nacional que hemos esbozado hasta aquí es donde se ubican las interrogantes que inspiran este trabajo y que conciernen al desarrollo de un campo abarcativo e interdisciplinar como es el de los Estudios Organizacionales. ¿Qué discusiones conlleva la conformación de algo así como los EO en Chile? ¿Qué factibilidad tiene la constitución del campo en el escenario académico del país? Estas preguntas se ubican en un terreno marcado por la diversificación de investigaciones y perspectivas disciplinarias que ponen al centro el interés por la organización y apelan a diversos actores a plantearse el desafío que acarrea la constitución de un campo de identidad incierta.

\section{Metodología}

Para la producción de datos, esta investigación se organizó siguiendo los procedimientos metodológicos del café comunitario o world café (Brown, \& Isaacs, 2005). 
Esta metodología es especialmente útil al momento de reunir una comunidad de participantes en torno a temáticas focalizadas en un tiempo limitado de conversación, permitiendo levantar perspectivas, opiniones y reflexiones a través de ciclos de sistematización temática y colectiva, llegando a una síntesis final de carácter gráfico y textual (Brown, 2002).

El desarrollo de esta metodología implica procesos de articulación, desarme y rearticulación de grupos heterogéneos de conversación en torno a preguntas dirigidas, de modo que a través de ciclos de intercambio secuencial se produce una polinización cruzada (Brown, \& Isaacs, 2005) de las conversaciones entre los diferentes participantes. Se trata de una metodología versátil en sus campos de aplicación según objetivos que recurrentemente apuntan al dialogo de comunidades y la reflexividad colectiva (Estacio, \& Karic, 2015; Fouché, \& Light, 2010). El caso de esta investigación y en coherencia con sus objetivos, las preguntas que guiaron la actividad fueron ¿Qué son los Estudios Organizacionales y cuál es su estado en Chile? ¿Cuáles serían sus oportunidades de desarrollo desde el ámbito académico?

Puesto que la polinización cruzada produce rearticulaciones de sentido como efecto de los procesos grupales de consenso y disenso que se movilizan en el diálogo generativo (Fouché, \& Light, 2010), se agregó una actividad preliminar en que se solicitó a los participantes responder a una pregunta individual y por escrito: ¿Qué son los Estudios Organizacionales para usted? Las diferentes respuestas a esta pregunta fueron igualmente consideradas para efectos de esta investigación.

Los participantes de este conversatorio fueron escogidos por medio de un muestreo intencionado y criterial (Palys, 2008), convocando a académicos de diferentes disciplinas y afiliaciones institucionales que contaran con una trayectoria de investigación y/o consultoría en organizaciones. De este modo, un criterio central del muestreo fue la expertise y/o trayectoria de los participantes en el campo de la investigación y enseñanza sobre organizaciones. Para su participación y previo al desarrollo de la actividad, se aplicaron consentimientos informados resguardando aspectos éticos de la investigación, incluyendo el anonimato y explicitando la utilización de los datos para la sistematización y análisis desarrollado en esta investigación.

Puesto que el campo de intervención e investigación en organizaciones posee un carácter multidisciplinario, durante el desarrollo de la actividad participaron académicos afiliados a departamentos de ingeniería, psicología, sociología, educación, economía y administración, así como profesionales ligados al mundo de la consultoría. Cabe destacar la heterogeneidad del grupo en términos de género, región de procedencia y trayectoria académica, además de una notoria divergencia entre la afiliación departamental de algunos y sus disciplinas de formación profesional. En total, incluyendo los coordinadores de la actividad, se sumaron 15 participantes en el world café.

La participación de los coordinadores entre los asistentes a la actividad se inspira en algunos de los lineamientos de la phronesis para la investigación organizacional (Flyvbjerg, 2004, 2006). Esto implica un recorrido por algunas interrogantes que refieren a la deseabilidad y la conveniencia de la investigación, en este caso movilizada por los valores explícitamente planteados por sus investigadores bajo de la forma de un posicionamiento frente al problema que anima la investigación, a saber, una disputa por los sentidos dominantes y la configuración académica de los estudios organizacionales en Chile. Esto se relaciona con la centralidad de las relaciones de poder que se juegan en este problema y han sido contextualizadas en los primeros apartados de este trabajo. Con ello, se abordan otras características de la phronesis en la investigación, entendida como la perspectiva ética y política que es asumida por quienes investigan al implicarse en una polifonía de voces (Flyvbjerg, 2004) movilizada por un diálogo primariamente generativo y secundariamente explicativo.

Para el análisis de los datos producidos se realizó una transcripción de los registros de audio correspondientes a cada uno de los ciclos de intercambio secuencial desarrollados por los diferentes grupos de conversación, así como las definiciones ofrecidas por cada participante. Luego se realizaron lecturas recursivas del contenido transcrito siguiendo las reglas del análisis de contenido temático (Vásquez, 1996). Esto permitió levantar categorías en resonancia con aspectos advertidos en la literatura sobre el desarrollo internacional de los EO, así como categorías que responden a elementos constitutivos del devenir de este campo en Chile. De este modo, los elementos centrales que surgieron son: la parcelación disciplinaria, la ambigüedad identitaria y las racionalidades en disputa.

\section{Resultados}

\section{Parcelación disciplinaria}

Uno de los primeros elementos que emergen a partir del análisis dice relación con la amplia geografía disciplinaria en que se ubica la producción de conocimiento y la enseñanza sobre las organizaciones. Junto con ser un elemento que se evidencia a partir de la composición de 
la muestra de participantes, el campo de estudio en torno a las organizaciones aterriza y se localiza en territorios disciplinares que no entran necesariamente en diálogo o que incluso divergen en la manera de concebir lo organizacional. Como señala una participante, "Hay otra pregunta que tienen que ver con la disciplina, (...) en qué disciplina estoy cuando miro el proceso de organizarse. Psicología, sociología, administración, economía, antropología" [Participante 1].

Los participantes coinciden en afirmar que el desarrollo del estudio en torno a las organizaciones se ha desplegado al interior de parcelas disciplinarias, de modo que las investigaciones que contribuyen a este campo lo hacen movilizando perspectivas parciales de referencia. Como afirma un participante:

'si uno revisa lo que se escribe generalmente en la academia, son escritos por psicólogos, o son escritos por comerciales, por sociólogos, por antropólogos, pero no se da mucho que sean escritos por personas desde ámbitos interdisciplinares' [Participante 2]

De este modo, las perspectivas de referencia responderían más a los encuadres disciplinares de quienes investigan que a un desconocimiento sobre la existencia de una perspectiva comprensiva y abarcativa como los EO, situación que se encuentra detrás de lo que hemos llegado a denominar como la diversidad paralizante que envuelve este campo.

En este sentido, puede afirmarse que existen fuertes identidades disciplinarias que hacen incómoda o inconveniente la adscripción de quienes investigan dentro del campo de los estudios organizacionales. Como señala una participante: "me cuesta ver el tema de las organizaciones como un ámbito de estudio propiamente tal, aislado. Me pasa que siempre he creído que es la dimensión organizacional la que uno debería tratar" [Participante 3]. Sin embargo, la difícil adscripción al campo coincide paradójicamente con el valor otorgado a la interdisciplinariedad al momento de definir lo que los participantes entienden por estudios organizacionales.

Esto resulta doblemente interesante si consideramos las apreciaciones que Ibarra-Colado, \& Montaño (1992) realizaron sobre la teoría organizacional hace más de 25 años, describiendo el campo como fragmentado por cuasi especialidades académicas sin comunicación ni tolerancia entre sus diferentes perspectivas teóricas, derivando en un estado de insuficiencias, simplezas y desafíos.

\section{Ambigüedad identitaria}

Junto a la parcelación disciplinaria como condición interviniente en el despliegue de los estudios organizacionales, un segundo elemento que surge es la ambigüedad identitaria del campo. Si bien existe claridad al momento de identificar los focos y objetos del estudio organizacional parceladamente orientado, no existe la misma claridad al momento de contextualizar la pregunta en un campo abierto, abarcativo e interdisciplinar. Ejemplo de ello sería la diversidad de focos atribuidos a este campo, incluyendo el estudio de individuos, grupos o relaciones, contextos institucionales e informales, escenarios productivos, gubernamentales y comunitarios, entre otros. Recuperando las preguntas movilizadas por una participante:

'¿Qué es lo que define el campo de estudio? ¿es el hecho que nos concentramos en las organizaciones como objeto? ¿eso es lo que queremos explicar? ¿o son las organizaciones como variable explicativa $o$ de comprensión de otros fenómenos?' [Participante 3].

La ambigüedad que reportan los intentos de definición de los estudios organizacionales entendidos como un campo interdisciplinar dice relación con el amplio espectro de fenómenos y materias abrazadas por él, aunque también con la diversidad de perspectivas que son convocadas para aproximarse a lo organizacional. Esto debido a que las organizaciones serían entendidas como un ente y también como un proceso, ambas orientadas desde múltiples propósitos. Como señala una participante:

'Si compartimos un fenómeno de estudio, un objeto de estudio, la pregunta es, ese fenómeno lo podemos leer de distintas perspectivas, de distintas disciplinas con distintos propósitos. Entonces, para mi, los estudios organizacionales si no tienen la capacidad de aceptar la diversidad, la heterogeneidad, los estudios funcionalistas, los críticos, los comprensivos, además de los sociológicos, los psicológicos, los económicos, los ecológicos, desde métodos cuanti y cuali, para mi esa es la gracia, la confluencia de esa heterogeneidad en el campo' [Participante 1]

Un último aspecto relacionado con la ambigüedad identitaria refiere a la invisibilidad de una comunidad académica que agrupe a quienes se adscriben a este campo y dónde pueda reconocerse quienes contribuyen a él, generan un conocimiento propio, proveen algún tipo de tradición o protagonizan sus acontecimientos fundacionales. En este sentido, no se reconoce la existencia de prácticas consistentes, convincentes e integradas sobre los estudios organizacionales, sino dispersión, segregación y aislamiento de diversos académicos que aportan parceladamente al estudio de las organizaciones.

Retomando la idea anterior, resulta significativo el alcance que realizan Sanabria, et al. (2013) respecto a los 
precursores internacionales de los Estudios Organizacionales quienes, en su momento, no reconocían lo que hacían bajo el nombre de Estudios Organizacionales puesto que la identificación del área fue posterior. Esto coincide con lo señalado por algunos de los contribuyentes a los estudios organizacionales en Chile quienes no se han sentido ni se sienten parte del campo: "yo no me adscribiría, nunca me he adscrito dentro de los estudios organizacionales (...) yo no estoy dentro del campo de los estudios organizacionales" [Participante 4].

En este sentido, los estudios organizacionales en el contexto chileno son percibidos como un campo en desarrollo y como un desafío contemporáneo para quienes se ubican en una generación académica que tiene la oportunidad de subvertir las condiciones disciplinarias que abonaron el surgimiento de la investigación y la enseñanza sobre las organizaciones en el país.

\section{Racionalidades en disputa}

El tercer elemento emergente a partir del análisis refiere a las racionalidades que disputan las prácticas de enseñanza, investigación e intervención en torno a lo organizacional. De este modo, al hablar de racionalidades nos referimos a un elemento que atraviesa las disciplinas que parceladamente se aproximan a lo organizacional, además de ser uno de los aspectos que explican la ambivalencia del campo y, por ende, la dificultosa identificación con él. Esta ambivalencia puede ser experimentada, siguiendo a Ibarra-Colado y Montaño (1992), como una suerte de pragmatismo que inmoviliza a quienes se acercan al campo organizacional cuando este ha sido cooptado por la racionalidad administrativa de corte irreflexivo. En palabras de una participante:

'para mi las organizaciones han sido un descubrimiento nuevo, necesario, pero siento que en el estudio de la sociología se ve con malos ojos de hecho, porque de alguna manera el campo se separa entre aquellos que ponen más énfasis en explicaciones estructurales y aquellos que tienen una perspectiva fenomenológica' [Participante 3]

Lo anterior dice relación con la difícil distinción entre los estudios organizacionales, de la administración y la gestión, considerando los contextos académicos en que dichos estudios se han territorializado, así como las perspectivas que han primado al hablar de cada uno de ellos. Como afirma una participante: "Yo creo que siempre que estás hablando de estudios organizacionales te estás topando con el management, ya sea de manera crítica o no" [participante 5]. En este sentido, existe la percepción que en Chile ha primado un enfoque administrativo con énfasis en la eficiencia y efectividad para dar respuesta a los diferentes escenarios en que el conocimiento y la expertise sobre lo organizacional son convocados:

'en Chile particularmente se ha metido un enfoque muy funcional, en donde queremos en realidad enseñar una determinada gestión, que sea eficaz, todo lo que es gestión estratégica de recursos humanos, gestión de comportamiento' [Participante 6].

La primacía del enfoque en la administración sugeriría que los estudios organizacionales, tal como se han desarrollado en el país, poseen un sesgo positivista, funcionalista y estratégico. Los temas con mayor reconocimiento en este campo serían el comportamiento organizacional, la gestión de personas y los recursos humanos (Guest, 1987; Reyes, 2016), mientras que sus contextos académicos privilegiados de producción, circulación y legitimación serían las escuelas de administración y negocios de las distintas universidades nacionales. Todo ello se relacionaría con la expansión y el dominio que posee esta corriente principal al haber devenido hegemónica en el trayecto del pensamiento organizacional en Chile, estigmatizando el campo y quienes dificultosamente se adscriben a él.

Es en contraposición a la hegemonía del enfoque administrativo donde se ubica el proyecto de los estudios organizacionales como campo interdisciplinario. A este último se le atribuiría una mayor diversidad teórica y metodológica, la reflexividad ético-política en torno a las prácticas organizacionales y una racionalidad menos técnica y más hermenéutico-crítica. Como señala una participante: “¿Qué es lo que entiendo como estudios organizacionales? Un enfoque comprensivo, a diferencia de los otros que son enfoques estratégicos y funcionales" [Participante 6].

De este modo, una concepción heterodoxa de lo organizacional contribuiría a legitimar materias comúnmente ignoradas o poco atendidas por el estudio disciplinar de las organizaciones, tales como el género, el poder, la subjetividad, la incertidumbre y la complejidad, en un ensayo de respuesta a una de las interrogantes movilizadas por uno de los participantes: "Tal vez la pregunta debiese ser ¿Qué tipo de estudios organizacionales queremos?" [Participante 7].

\section{Discusión}

A partir de los análisis realizados puede afirmarse que el campo académico de los EO en Chile se encuentra en un estado incipiente y en desarrollo, apareciendo como un campo ambiguo y atravesado por la parcelación 
disciplinar. Estos aspectos, que emergen desde una polifonía de voces convocadas a la reflexión ético-política movilizada por el dialogo generativo (Flyvbjerg, 2004), ofrecen un conjunto de pistas sobre las circunstancias que mediarían en la configuración académica de los estudios organizacionales en Chile. En este sentido, se trata de aspectos que pueden ser esperables durante la gestación de cualquier campo de estudio interdisciplinario (tal como ocurrió con los estudios culturales o los estudios de género), lo que no nos exime de explorar la relación que estos guardan con la trayectoria del pensamiento organizacional chileno, las especificidades que dicha trayectoria imprime a su devenir y las interrogantes que habilita la discusión de estos aspectos para intencionar y desear nuevos derroteros para el pensamiento organizacional en Chile.

De este modo y a la luz de estos resultados resulta conveniente un retorno al devenir histórico del pensamiento organizacional en el país, toda vez que en él pueden rastrearse las transformaciones que marcaron su trayectoria hasta la actualidad. En este sentido, las particularidades de la Dictadura en Chile constituyen un punto de inflexión y referencia ineludible para comprender la clausura radical de la heterodoxia organizacional, así como la marginación de las corrientes más críticas, las que fueron explícitamente vigiladas, perseguidas y amenazadas por el régimen y sus intervenciones en la academia, especialmente en las ciencias sociales. Los resabios de este periodo se resienten hasta nuestros días y son constatables en aquella cultura del enemigo (Heine, 2002) que inicialmente se experimentó como vigilancia epistémicopolítica sobre la academia $^{2}$ y hoy perdura en la bifurcación de disciplinas y racionalidades, paradigmáticamente expresadas en una polarización entre las ciencias sociales y el mundo de la administración y las empresas.

Es en relación a lo anterior que emerge otro aspecto significativo, a saber, el dominio que en el campo organizacional chileno ejercen tanto el paradigma de los recursos humanos como la racionalidad funcionalista asociada a la gestión y la administración. En este sentido, los EO serían valorados por su interdisciplinariedad, ofreciendo una perspectiva amplia y abarcativa para renovar la enseñanza, la investigación y la intervención contemporánea en torno al fenómeno organizacional.

En este contexto, los desafíos que hemos retratado se anudan en una paradoja. Junto a la poca claridad sobre la identidad de este campo existe una dificultosa

\footnotetext{
2 Ejemplos ilustrativos a este respecto son la 'caza de brujas' de académicos disidentes al modelo neoliberal en escuelas de economía y administración, así como el cierre de departamentos de sociología,
}

adscripción a él, lo que depende de los escenarios académicos, disciplinares y profesionales de quienes declaran esta complejidad. Mientras que en escenarios ligados a la administración, los estudios organizacionales parecerían portar una mirada crítica que estigmatiza a sus cultores, la misma adscripción al campo en escenarios de ciencias sociales se experimenta como fuente de estigma, esta vez por suponerse que el estudio de las organizaciones se reduce a una mirada funcionalista y equiparable a la administración o la gestión de recursos humanos.

Como se evidencia a nivel internacional, existe una profusa producción en torno a los estudios organizacionales que da cuenta de la construcción de un campo de desarrollo académico heterogéneo, controversial y rico en matices, que ha logrado significativos niveles de consolidación como un campo de estudio y que ha encontrado domicilio, dentro y fuera de Latinoamérica, en diversas redes y asociaciones que sustentan publicaciones y encuentros académicos. En contraste, en Chile el campo es incipiente y fragmentario, dadas las condiciones y desafíos que hemos esquematizado en este artículo. A nivel de enseñanza y práctica, esto puede traducirse en una mayoritaria importación de teoría y modelos organizacionales, invisibilizando las parceladas o inadvertidas producciones locales en el campo y sus potencialidades emancipatorias y decoloniales (Faria, 2015; Misoczky, 2017).

Expuesto lo anterior, resulta pertinente y oportuno ensayar una formación sobre los EO que haga sentido para la realidad chilena, considerando el devenir local del pensamiento organizacional y sus comunidades de práctica dentro y fuera de la academia. A este respecto, sostenemos que los EO constituirían una oportunidad para disputar la racionalidad dominante -o lo que hemos denominado como corriente principal- que ha hegemonizado el pensamiento organizacional en Chile a lo largo de los últimos 30 años. Así, la pregunta por un campo como los EO permite activar reflexiones no solo disciplinarias, sino éticas y políticas respecto a las prácticas que se dan en la vida organizacional, a propósito de los aportes que perspectivas críticas y heterodoxas conllevan cuando son legitimadas en este campo.

Lo anterior adquiere especial sentido toda vez que los EO podrían contribuir a la construcción de un acervo propio y situado en teoría organizacional, que dispute la promoción, legitimidad, privilegios y financiamiento alrededor del área de administración (Cooke, \& Faría, 2013). Sin embargo, esto abre un dilema respecto a la

cuando no su intervención por militares durante la dictadura de Augusto Pinochet, algo experimentado igualmente por otras áreas de las ciencias sociales. 
institucionalización de los EO, un campo que se debate entre la afirmación, la oposición o incluso la permanente indefinición. Mientras la delimitación, institucionalización y definición del campo podrían rigidizar su amplitud y dinámica, la no delimitación podría opacar las oportunidades críticas que este ofrece, manteniendo la hegemonía existente de las perspectivas funcionalistas.

Finalmente cabe regresar sobre la pertinencia y relevancia que potencialmente el desarrollo de un campo como los EO podría ofrecer como respuesta al status quo económico y social. En un escenario de profundas y aceleradas transformaciones socioculturales $y$ económicas, globales y locales, que afectan al mundo del trabajo y las organizaciones, la emergencia de un campo interdisciplinario podría contribuir a aprehender de mejor forma fenómenos complejos como las migraciones, la desigualdad de género y el cambio climático, por solo mencionar algunos. En este contexto, la distinción entre administración y organización vuelve a la superficie, donde la racionalidad de la primera ha ejercido un dominio sobre la segunda. Un campo como los EO, con las consideraciones que hemos revisado, podría proponer una alternativa crítica frente a las formas de adaptación del capitalismo (Boltanski, \& Chiapello, 2002) y sus estrategias de adhesividad afectiva a la ideología managerial. Lo anterior, reactivando algunas preguntas que consideramos centrales para este campo: ¿Quién organiza y quién define los objetivos de dicha práctica? ¿Para qué nos organizamos?

\section{Referencias}

Abarca, N., Majluf, N., \& Rodríguez, D. (1998) Identifying Management in Chile. International Studies of Management \& Organization, 28 (2), 18-37, DOI: http://dx.doi.org/10.1080/00208825.1998.11656 $\underline{732}$

Acuña, A. (2012). Management Flexible y Toxicidad Organizacional: Socio-análisis de una novela chilena. PRAXIS. Revista de Psicología, 14(21), 1133.

Acuña, E. (2017). Estudios organizacionales en la Facultad de Economía y Negocios (FEN), Universidad de Chile (1958-2017): Referencias históricas. Presentación en seminario “¿Estudios organizacionales en Chile? Oportunidades, perspectivas y proyecciones", 15 de mayo 2017, Universidad Diego Portales).

Acuña, E., \& Silva, C. (2008). Malestar en Organizaciones Chilenas: Historias de Trabajadores. Revista de Psiquiatría y Salud Mental, 25 (1-2), 105-119.

Acuña, E., \& Sanfuentes, M. (2011). Institutional abuse:
Caught between professional vocation and system's efficiency. En L. Gould, A. Lucey, y L. Stapley (Eds.). The reflective citizen: Organizational and social dynamics (pp. 111-129). London, UK: Karnac

Acuña, E., \& Sanfuentes, M. (2016). Ambiguity in the Identity transformation of Public Health Organizations. RAE-Revista de Administração de Empresas , 56 (3), 330-341. doi:10.1590/\$0034756039020106

Ascorra, P. (2003). La organización como sistema discursivo: un análisis de caso en una universidad chilena (Tesis de Doctorado). Facultad de Ciencias Sociales, Universidad de Chile, Santiago

Assaél, J., Cornejo, R., González, J., Redondo, J., Sánchez, R., \& Sobarzo, M. (2011). La empresa educativa chilena. Educaçao \& Sociedade, 32 (115), 305-322

Barba, A. (2012). Los estudios organizacionales. Una aproximación al caso de México. Estudios Interdisciplinarios de La Organización, 1(1), 4-23

Barba, A., \& Ramírez, G. (2004). El posgrado en estudios organizacionales: programas, redes y vinculación. Omnia, 20, 275-280.

Boltanski, L., \& Chiapello, È. (2002). El nuevo espíritu del capitalismo. Madrid: Akal

Brown, J. (2002). The World Café: A Resource Guide for Hosting Conversations That Matter. Mill Valley, CA: Whole Systems Associates.

Brown, J., \& Isaacs, D. (2005). The World Cafe. Shaping Our Futures through Conversations That Matter. San Francisco: Berrett-Koehler.

Chumbita, H. (2015). Fundamentos para los estudios organizacionales: aportes del pensamiento crítico suramericano. Cadernos EBAPE. BR, 13(2), 390401.

Cooke, B., \& Faría, A. (2013) Editorial. Development, Management and North Atlantic Imperialism: For Eduardo Ibarra Colado. Cadernos EBAPE. BR, 11(2), 1-14.

de Faria, J. H. (2009). Teoria crítica em estudos organizacionais no Brasil: O estado da arte. Cadernos EBAPE.BR 7(3), 509-515.

Espinoza, J.F., \& Rivera, G. (2015). Los Estudios Críticos de la Administración en Chile [Archivo PDF]. Recuperado de http://www.criticalmanagement.org/node/3206

Estacio, E.V., \& Karic, T. (2015) The World Café: An innovative method to facilitate reflections on internationalisation in higher education. Journal of Further and Higher Education, 40(6), 731-745.

Fardella, C., Sisto, V., \& Jiménez, F. (2017). La transformación de la universidad y los dispositivos de cuantificación. Estudos de Psicologia (Campinas), 34(3), 435-448. https://dx.doi.org/10.1590/1982- 


\section{1}

Faría, A. (2016). Management 'global' y de/colonialidad. Conferencia ofrecida en seminario "Desorganizar y reorganizar: Miradas críticas y alternativas en teoría organizacional", 4 de junio 2015, Universidad Diego Portales.

Flyvbjerg, B. (2004). Phronetic Planning Research: Theoretical and Methodological Reflections. Planning Theory \& Practice, 5 (3), 283-306

Flyvbjerg, B. (2006). Making Organization Research Matter: Power, Values and Phronesis. En S. Clegg, C. Hardy, T. S. Lawrence y W. Nord, (Eds.), The Sage handbook of organization studies (pp. 370387) Thousand Oaks, CA.: SAGE.

Foladori, H., \& Guerrero, P. (Eds.). (2018). Malestar en el trabajo. Desarrollo e intervención. Santiago: LOMFouché, C., \& Light, G. (2010). An invitation to dialogue. The world café in social work. Qualitative social work, 10(1), 28-48.

Garretón, M.A. (2013) Cuarenta años después del golpe de Estado. Diálogo Global, 3 (5), 4-7.

Gómez, C., \& Rodríguez, J. (2006). Four approximations keys to Chilean culture: Authoritarianism, legalism, fatalism and compadrazgo. Asian Journal of Latin American Studies, 19(3), 43-65.

Gonzales-Miranda, D. R. (2014). Los estudios organizacionales. Un campo de conocimiento comprensivo para el estudio de las organizaciones. Innovar, 24(54), 43-58.

Gonzales-Miranda, D. R., Ocampo-Salazar, C.A., \& Gentilin, M. (2018). Organizational Studies in Latin America. A Literature Review (2000-2014). Innovar, 28(67), 89-109. http://dx.doi.org/10.15446/innovar.v27n67.6861 $\underline{5}$

Guest, D. (1987) Human Resource Management and Industrial Relations. Journal of Management Studies, 24, 503-521

Hax, A., \& Majluf, N. (1996). Gestión de Empresa con una Visión Estratégica. Santiago de Chile: Dolmen

Heine, J. (2002). Desarrollo Humano en Chile 2002. Nosotros los chilenos: un desafío cultural. Perspectivas en política, economía y gestión, 6 (1), 165-173.

Hojman, D., \& Pérez Arrau, G. (2005). Cultura nacional y cultura organizacional en tiempos de cambio: la experiencia chilena. Revista Latinoamericana de Administración, 35, 87-105. Ibarra-Colado, E., \& Montaño, L. (1987). Orden organizacional: poder, estrategia y contradicción. México: UAM-I.

Ibarra-Colado, E., \& Montaño, L. (1989). Teoría de la organización. Fundamentos y controversias. México, Universidad Autónoma Metropolitana.

Ibarra-Colado, E., \& Montaño, L. (1991). Ensayos críticos para el estudio de las organizaciones en México.
México: Porrúa/UAM-I. Ibarra-Colado, E., \& Montaño, L. (1992). Mito y poder en las organizaciones. Un análisis crítico de la teoría de la organización. México: Trillas.

Ibarra-Colado, E. (2006). Organization studies and epistemic coloniality in Latin America: Thinking otherness from the margins. Organization 13(4), 463-488. doi: 10.1177/1350508406065851

Imas, J. M. (2014). Rational Darkness: Voicing the Unheard in The Modern Management Discourse of Chile, Administrative Theory \& Praxis, 27(1), 111-133

Johansen, O. (1971). Sistemas y Organizaciones. Santiago de Chile: INSORA, 1971

Johansen, O. (1980). Anatomía de la empresa : una teoría general de las organizaciones sociales. Santiago de Chile: Facultad de Ciencias Económicas y Administrativas, Departamento de Administración, Universidad de Chile

Johansen, O. (1989). La Administración Como Fenómeno Natural. Santiago de Chile: Facultad de Ciencias Económicas y Administrativas, Universidad de Chile

Johansen, O., de la Cuadra, R., \& García, V. (1992). Hacia una teoría de la organización. Santiago, Chile: Editorial de Economía y Administración

Julián, D. (2018a). Precariedad laboral y estrategias sindicales en el neoliberalismo: Cambios en la politización del trabajo en Chile. Psicoperspectivas, 17(1). https://doi.org/10.5027/psicoperspectivas-vol17issue1-fulltext-947

Julián, D. (2018b). Características organizacionales del sindicalismo en Chile: Investigación Acción en sindicatos de la Región de la Araucanía. Revista Mexicana de Ciencias Políticas y Sociales, 63(232), 269-300. https://doi.org/10.22201/fcpys.2448492xe.2018. 232.58312

Majluf, N. (2011). Los desafíos de la gestión. De lo formal a lo sutil. Santiago de Chile: Aguilar

Mandiola, M. (2010). Latin America's Critical Management? A Liberation Genealogy. Critical Perspectives on International Business, 6 (2), 162176.

Mandiola, M. (2013). Management education in Chile: From politics of pragmatism to (im)possibilities of resistance. Universitas Psychologica, 12(4), 10871100Mandiola, M. (2017). Ambición. Gestión y $\begin{array}{llll}\text { Tendencias, } & 2 & \text { (3), }\end{array}$ http://dx.doi.org/10.11565/gesten.v2i3.46

Mandiola, M. (2018) La mirada decolonial al management. Gestión y Tendencias, 3(1), 5-10.

Mandiola, M., \& Ascorra, P. (2010). Chilean management education: rhetoric of pragmatism, consumerism, 
individualism and elitism. Cadernos EBAPE.BR, $8(2), 370-387$.

Mandiola, M., Imas M., \& Ascorra P. (2014). Cambios y transformaciones en el ejercicio del Management. Psicoperspectivas. Individuo y sociedad, 13 (3), 15.

Mandiola, M., \& Varas, A. (2016). Un asunto menor, administrativo: la universidad y el management. Gestión y Tendencias, 1(4), 2-5, http://dx.doi.org/10.11565/gesten.v1i4.25

March, J. G. (2007). The study of organizations and organizing since 1945. Organization Studies, 28 (1), 9-19.

Medina, C. (2010). Los estudios organizacionales entre la unidad y la fragmentación. Cinta Moebio, (38), 91109

Misoczky, M. (2017). ¿De qué hablamos cuando decimos crítica en los estudios organizacionales?. Administración \& Desarrollo, 47(1), 141-149.

Moulian, T. (1997).Chile actual: Anatomía de un mito. Santiago de Chile: LOM

Nicholls-Nixon, C., Davila, J., Sanchez, J., \& Rivera, M. (2011). Latin America Management Research: Review, Synthesis, and Extension, Journal of Management, 37 (4): 1178-1227.

Özbilgin, M. (2004). 'International' Human Resource Management: Academic Parochialism in Editorial Boards of the 'Top' 22 Journals on International Human Resource Management. Personnel Review, 33 (2), 205-221

Padilla, A.; Hernández, C., \& Ríos, R. (2015). Los estudios organizacionales en América Latina: un diagnóstico con base en la difusión del campo de estudio en Colombia, Brasil y México. Disponible en

http://congreso.investiga.fca.unam.mx/docs/xx/d ocs/17.08.pdf

Palys, T. (2008). Purposive sampling. En L. M. Given (Ed.) The Sage Encyclopedia of Qualitative Research Methods. (Vol.2). (pp. 697-698). Los Angeles: SAGE.

Pérez-Arrau, G., Eades, E., \& Wilson J. (2012). Managing human resources in the Latin American context: the case of Chile. The International Journal of Human Resource Management, 23(15), 31333150.

http://dx.doi.org/10.1080/09585192.2011.63954 $\underline{7}$

Ramírez, L. (2003). Hacia un análisis Crítico de la Gestión: presentando los Critical Management Studies. Revista Chilena de Administración Pública, 2(4), 7 25.

Ramírez-Martínez, G., Vargas, G., \& De la Rosa, A. (2011). Estudios Organizacionales y administración. Contrastes y complementariedades. Caminando hacia el eslabón perdido. Forum Doctoral, 3(3), 754.

Ramos, C. (2009). La transformación de la empresa chilena. Una modernización desbalanceada. Santiago, Chile: Universidad Alberto Hurtado

Reed, M. (2017). Teorización organizacional: campo históricamente controvertido. En G. Ramírez y D. González-Miranda (Eds.) Tratado de estudios organizacionales. Vol. 1: Teorización sobre el campo (pp. 41-78). Medellín, Colombia: SAGE, Universidad EAFIT

Reyes, G. (2016). Historia y desarrollo de la disciplina académica de R.R.H.H. 1973-1990 (tesis de grado), Universidad de Chile, Santiago, Chile.

Ríos, N., Mandiola, M., \& Varas, A. (2017) Haciendo género, haciendo academia. Un análisis feminista de la organización del trabajo académico en Chile. Psicoperspectivas, 16(2), 114-124. http://dx.doi.org/10.5027/psicoperspectivasvol16-issue2-fulltext-1041

Rodríguez, D., \& Arnold, M. (1991). Sociedad y teoría de sistemas. Santiago: Editorial Universitaria.

Rodríguez, D. (1991). Gestión organizacional: elementos para su estudio. Santiago, Chile: Universidad Católica de Chile.

Rodríguez, D. (1992). Diagnóstico Organizacional. Santiago: Ediciones Universidad Católica de Chile

Rodríguez, D. (2004). Organizaciones para la modernización. México, D.F.: Universidad Iberoamericana.

Rodríguez, D., \& Opazo, M. P. (2007). Comunicaciones de la organización. Santiago, Chile: Ediciones Universidad Católica.

Rodríguez, D., Bozzo, C., \& Arnold, M. (1999). Cultura organizacional e innovación: El caso de GoodyearChile. En: A. Dávila, \& N. Martínez, (Eds.), Cultura en organizaciones latinas (pp.71-116). México, D. F.: Siglo Veintiuno.

Rodríguez, J. K., \& Gómez, C.F. (2009). HRM in Chile: The impact of organisational culture. Employee Relations, 31(3), 273-294. http://dxdoi.org/10.1108/01425450910946479

Salgado, C. (2007). ¿Qué son los estudios organizacionales? Revista Universidad EAFIT, 43(148), 9-24.

Sanabria, M., Saavedra, J., \& Smida, A. (2013). Los estudios organizacionales ("Organization studies"): Fundamentos, evolución y estado actual del campo. Bogotá, Colombia: Editorial Universidad del Rosario

Sanfuentes, M., \& Acuña, E. (2014). The castaways of life: A study of organizational remembering within the context of a Chilean geriatric hospital. Culture and Organization, 20(4), 288-306.

http://dx.doi.org/10.1080/14759551.2013.87264 
$\underline{3}$

Sisto, V. (2004). Teoría(s) Organizacional(es) postmoderna(s) y la gest(ac)ión del sujeto postmoderno. Barcelona, España: Universitat Autònoma de Barcelona-TDX. Disponible en http://www.tdx.cesca.es/TDX-1217104-143200/ (ISBN 84-688-7772-7)

Sisto, V. (2005). Flexibilización laboral de la docencia universitaria y la gestación de la universidad sin órganos: Un análisis desde la subjetividad laboral del docente en condiciones de precariedad. En P. Gentili, \& B. Levy, (Eds.), Espacio público y privatización del conocimiento. Estudios sobre políticas universitarias en América Latina (pp. 523574). Buenos Aires, Argentina: CLACSO.

Soto, A. (Ed.). (2008). Flexibilidad laboral y subjetividades. Santiago, Chile: LOM.

Soto, A. (2014). Escenarios del trabajo en Chile: Una propuesta comprensiva de la actividad laboral. En: A. Stecher, \& L. Godoy, (Eds.), Transformaciones del trabajo, subjetividad e Identidades (pp.299322). Santiago, Chile: RIL.
Stecher, A. (2015). El campo de investigación sobre transformaciones del trabajo, identidad $y$ subjetividad en la modernidad contemporánea. Apuntes desde Chile y América Latina. En: L. Godoy, \& A. Stecher, (Eds.), Transformaciones del Trabajo, subjetividad e Identidades. (pp.19-32). Santiago: RIL.

Stecher, A., \& Godoy, L. (2014). Transformaciones del trabajo, subjetividad e identidades. Santiago de Chile: RIL

Subiabre, J. P. (2012). Educación en Management en una Escuela de Negocios de Chile: Análisis desde la experiencia personal y desde enfoques críticos (tesis de grado), Universidad de Chile, Santiago, Chile.

Vásquez, F. (1996). El análisis de contenido temático. Objetivos y medios en la investigación psicosocial. (Documento de trabajo) (pp. 47-77). Barcelona, España: Universitat Autònoma de Barcelona.

Zabala, X., Guerrero, P., \& Besoain, C. (2017). Clínicas del trabajo: Aproximaciones teóricas y estrategias de Intervención. Santiago, Chile: Ediciones Alberto Hurtado. 